\title{
Design and Simulation of High-voltage Power Supply for Electron Beam Precision Micro Machining Equipment
}

\author{
Xuejiao $\mathrm{Li}^{1, \text { a }}$, Shouqi Wei ${ }^{1, \mathrm{~b}}$, Xian Luo ${ }^{2, \mathrm{c}}$,Jinpeng Zhu ${ }^{1, \mathrm{~d}}$, and Weipeng Yin ${ }^{1, \mathrm{e}}$ \\ ${ }^{1}$ Guilin University of Electronic Technology, Guilin 541004, China; \\ ${ }^{2}$ Authorities Affairs Bureau of Mashan County, Mashan 530600, China.

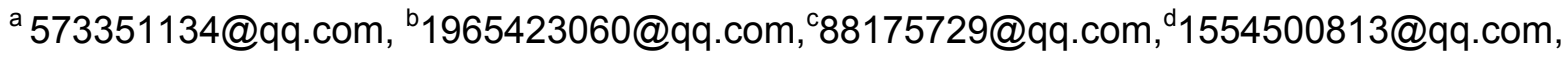 \\ e1275788220@qq.com
}

Keywords: electron beam, precision micro machining, pressure sensitive resistance, intermediate frequency inverter, high-voltage supply

\begin{abstract}
A kind of high-voltage inverter power supply for electron beam precision micro machining equipment is introduced. The system is based on the current transformation way of $\mathrm{AC} \rightarrow \mathrm{DC} \rightarrow \mathrm{AC} \rightarrow \mathrm{AC} \rightarrow \mathrm{DC}$. Its intermediate inverter link is intermediate frequency inverter which is composed of two phase parallel half-bridge inverter, this can solve the problem of discontinuous current when is in light load. Because it is medium frequency inverter, the realization of energy transfer is intermediate frequency, so the cost of the equipment is reduced. In the high-voltage side, the over-voltage can be effectively suppressed by using the pressure sensitive resistance. The mathematical model of the inverter high-voltage power supply is built by the simulation software PSIM. The simulation results show that the performance of the power supply can meet the expected design target and guarantee the welding quality of the EBM.
\end{abstract}

\section{Introduction}

Precision micro machining technology is seeking to achieve the highest accuracy and the size limit of processing technology which is represented with precision engineering, micro engineering and nano technology. Precision micro machining technology is one of the important signs to measure the overall level of a country's manufacturing industry. It not only determines the machining accuracy and surface quality of mechanical products, but also reflects the level of cutting-edge technology and national defense industry. Electron beam machining (EBM) is a new kind of special processing technology got rapid development in recent years, it is a kind of high-tech product which integrates many kinds of technologies, such as vacuum physics, electronic technology, electronic optics, high voltage technology, computer and control technology. EBM is using high energy density of electron beam to carry out drilling, cutting and welding, lithography and other work, it is not only a kind of precision and ultra precision machining method, but also a kind of important micro machining method.

The accelerate high-voltage supply of EBM electron gun is mainly to provide acceleration voltage for electron gun, whose performance directly decides the welding process and quality of EBM. It is one of the key components of the EBM, and its reliability is very important to the whole equipment. In practical work, it is found that stability and ripple of power supply have direct influence on the magnitude of the beam, the diameter of the beam, the position of the beam, the change of the beam current, the size of the scanning field and the sensitivity of the deflection. These factors affect the micronization of processing for the electron beam precision micro machining equipment in the practical work. So it's very important for the power supply to ensure that it can have the characteristics of small output voltage ripple coefficient and high stability. A kind of square wave inverter high-voltage power supply for electron beam precision micro machining equipment is described in this paper. 


\section{The main circuit structure and working principle of high-voltage power supply}

High-voltage Power Supply. The process of realizing the transformation of current energy for the designed high-voltage power supply is: network voltage input(AC) $\rightarrow$ low voltage rectifier filter (DC) $\rightarrow$ intermediate frequency square wave inverter(AC) $\rightarrow$ isolating step-up transformer (AC) $\rightarrow$ high voltage rectifier filter(DC) $\rightarrow$ high-voltage protection resistance(DC). At the same time, by setting the high voltage sampling signal and the beam sampling signal as negative feedback to the PI regulator, the duty ratio of the pulse width modulation (PWM) output wave is controlled, and then the inverter can be adjusted to provide a stable power supply for the electron beam precision micro machining equipment. Structure diagram of EBM power supply is shown in figure 1.

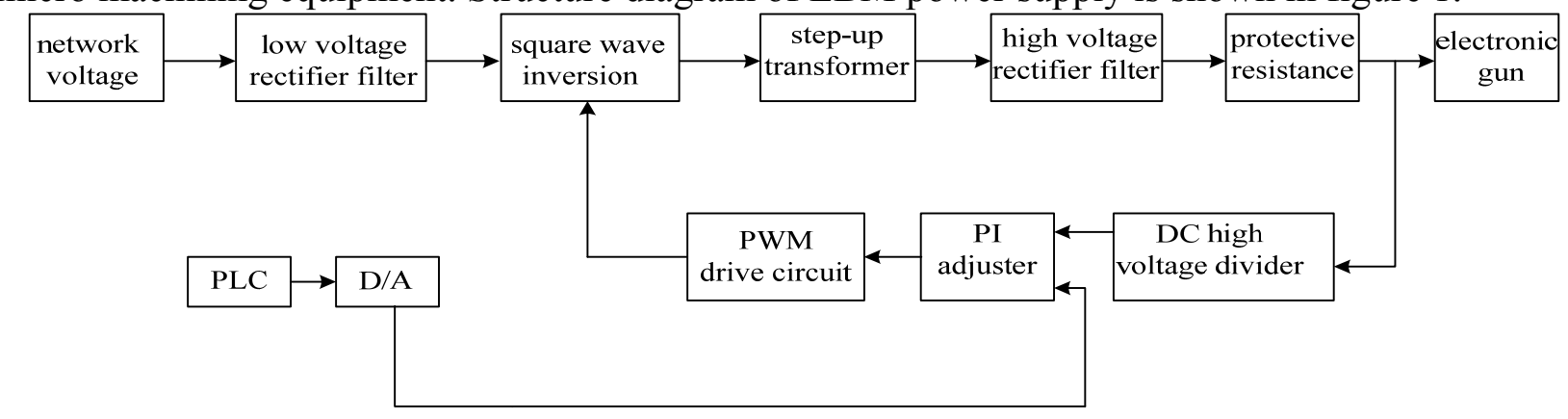

Fig.1 Structure diagram of EBM power supply

As the system set intermediate frequency AC which is produced by two-phase half-bridge inverter into the intermediate frequency isolation high-voltage transformer, and then through the high voltage rectifier filter, high-voltage protection resistance, high-voltage discharge choke circuit to get high-voltage output. It has the characters of small ripple coefficient and steady operation .The traits of this design are the use of intermediate frequency square wave inverter, intermediate frequency booster, also using voltage dependent resistor(VDR) as high-voltage stabilizer. The two-phase parallel half-bridge medium frequency inverter which is based on bipolar PWM can solve the problem of current discontinuous when is at light load, and then restrain spike pulse. The outstanding advantage of square wave is that the output coefficient is big and the waveform is smooth, using square wave voltage which is obtained by the intermediate frequency inverter as primary voltage of the boost transformer can not only ensure good performance for the high-voltage power supply ,but also make the manufacturing process of the step-up transformer more simple. It can reduce the manufacturing cost. So the system can filter out the AC components and meet the ripple requirements when it's elected to a smaller high voltage filter capacitor. The smaller filter capacitance is helpful to improve regulating speed of the control system and reduce the impact of the high-voltage discharge because of its small storage ,thus to improve the reliability of the power supply system. At the high-voltage side, the VDR can be used as a voltage stabilizer to suppress the transient over-voltage, this can avoid the damage to the high voltage power supply, ensure the normal operation and prolong the service life.

Low-voltage rectifier filter. Three-phase network voltage is $380 \mathrm{~V} \pm 10 \%, 50 \mathrm{~Hz}$. Because of the network voltage is send to the square wave inverter after the treatment of rectification and filtration, so it is not suitable to use more complex controlled rectifier. This design uses a three-phase full wave uncontrolled rectifier. After three-phase rectifier, the output is pulsating DC, and the average value is $1.35 \mathrm{U}$, that is $514 \mathrm{~V}$. But as the voltage fluctuation is very big, it need to filter and the output voltage is $532 \mathrm{~V}$.

Intermediate frequency square wave inverter. This square wave inverter is a new type of two-phase parallel half-bridge medium frequency inverter which is based on bipolar PWM and using IGBT as power switching device. The driver waveforms of the two sets of inverter bridge are high frequency bipolar PWM driver waveforms of 90 degrees phase differences. The drive signals for each group's upper and lower half bridge power tubes are complementary, but the conversion process is equipped with corresponding die area to avoid the power tubes straight through in a short period of time. The output intermediate frequency square wave of the two sets are of 90 degrees phase differences and the phase sequence distribution is symmetrical. The main 
function of the inverter is that the inverter makes the uncontrolled DC into intermediate frequency $\mathrm{AC}$ square wave by the high frequency PWM.

Intermediate frequency step-up transformer. The boost transformer is mainly used to realize the transmission of electric energy, the transformation of the voltage value and the high voltage insulation. As the design realizes the medium frequency energy transfer, the manufacturing process is simpler and the manufacturing cost is reduced.

High-voltage rectifier filter. High-voltage rectifier still use a relatively simple uncontrolled rectifier. High-voltage filter adopts $\pi$ type LC filter circuit. The difference between the EBM filter circuit and the conventional filter circuit is that the filter capacitor of EBM is smaller and the filter inductor of EBM is larger.

\section{Control circuit}

The system is controlled by current-voltage closed-loop control strategy in this paper. when it's functioning properly, comparison the high-voltage given signal with the sampling signal to use in PI regulator, then the duty ratio of the PWM output wave is adjusted by the PI regulation to control the output of the accelerating voltage. So the system can achieve the accuracy of the output voltage and ensure the purpose of stability for the accelerating power supply.

\section{Protective circuit}

The power supply maybe will have over-voltage and over-current when it's functioning, thereby affect the normal work of the EBM. The characteristic of this design is using VDR as high voltage regulator components, it is parallel with the high voltage rectifier filter. The VDR has the characteristics of good nonlinearity, low residual voltage, rapid action, small leakage current and large discharge current capacity. Although it has been widely used in the protection of power industry, the study on the VDR in the high-voltage power supply of the EBM is rare. When the system operates normally, the voltage across VDR is rated voltage which is less than it's operation voltage. When there is over-voltage across the VDR, it will quickly move into voltage limiting state and make the voltage be clamped on a relatively fixed value. In this process, the voltage value of the VDR is greater than it's action voltage value. If the current exceeds the current capacity, the VDR may be damaged due to overheating, so a current sampling resistor is in series with the VDR. The high-voltage protection circuit is shown in Figure 5, $\mathrm{R}_{\mathrm{y} 1}$ is the VDR, $\mathrm{R}_{1}$ is the current sampling resistor.

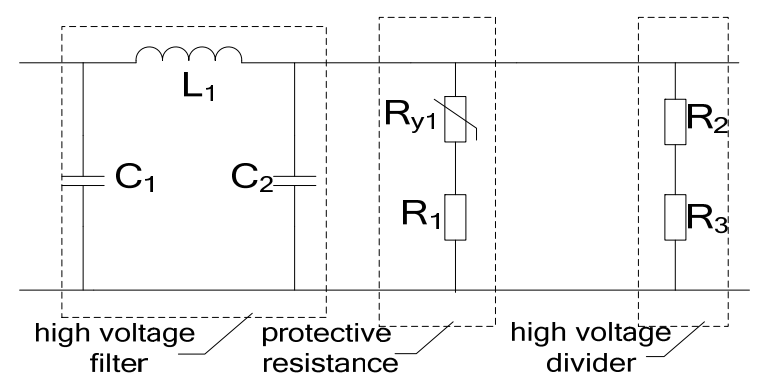

Fig.2 High-voltage protection circuit

\section{The Simulation and the Analysis of the High-voltage Power Supply System}

Simulation System. To validate the the feasibility and advantage of the proposed high-voltage inverter power supply for electron beam precision micro machining equipment, the whole high-voltage power supply is modeled by using the software PSIM. We will emphatically analyze the inverter part.

The technical index of the power supply system:

Input voltage: $380,50 \mathrm{~Hz}$

Output voltage :0 110KV, ripple coefficient is less than $1 \%$, stability is $10^{-4}$ 
Electron beam: $0 \sim 10 \mathrm{~mA}$, ripple coefficient is less than $1 \%$

Efficiency: $>80 \%$

Analysis of the Simulation.Figure 3 shows the waveforms of the high-voltage power supply when it's running at light load. V20,V21,V10,V9 are respectively the drive signals of the upper and lower IGBT for the two sets of inverter. The voltage $u_{\mathrm{a} 1}, \mathrm{u}_{\mathrm{b} 1}$ are primary side input voltages of the step-up transformers, $\mathrm{i}_{\mathrm{a} 1}, \mathrm{i}_{\mathrm{b} 1}$ are inductor current. We can see the inductance current is still continuous, this can solve the problem of current discontinuous. The simulation waveform is consistent with theoretical waveform.
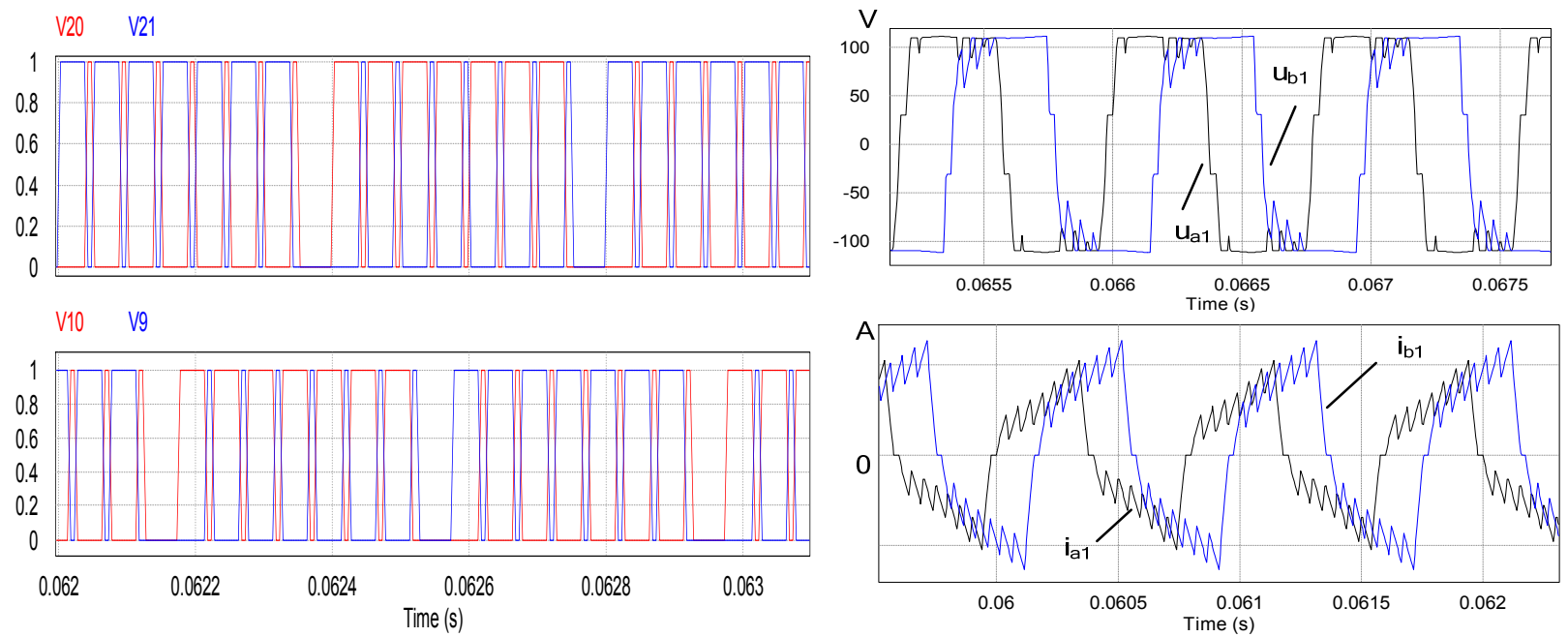

Fig.3 Drive signals of inverter, simulation waveform of $u_{a 1}, u_{b 1}, i_{a 1}$ and $i_{b 1}$ when power supply is running at light load

Figure 4 shows the output voltage $U_{O}$ of the power supply which has VDR as its protective resistence. We can see $U_{O}$ is clamped in $110 \mathrm{KV}$ by VDR. This can effectively suppress overvoltage.
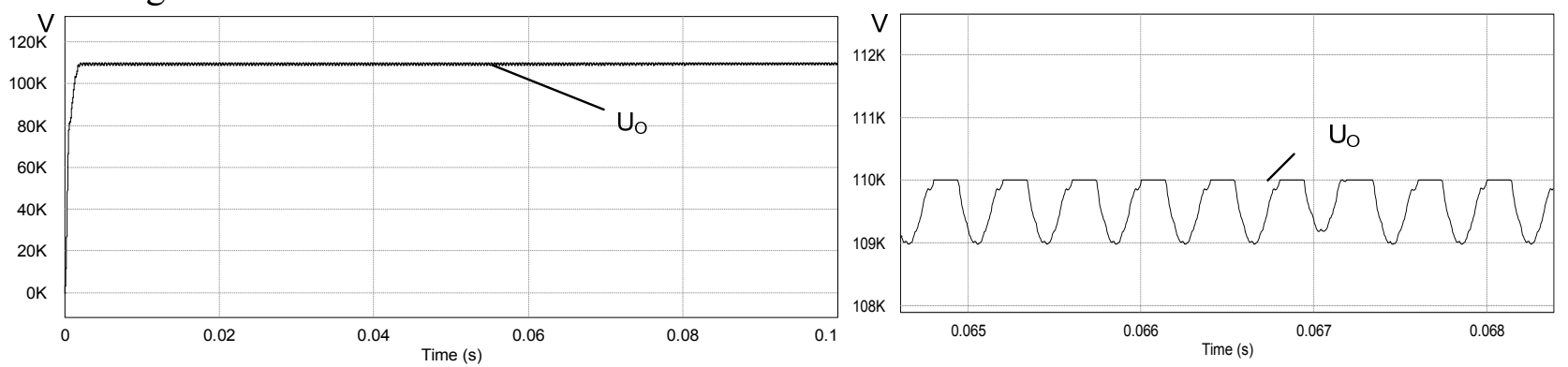

Fig.4 The output voltage $U_{O}$ of the power supply

\section{Conclusions}

A new kind of high-voltage power supply for electron beam precision micro machining equipment is introduced in this paper. Theoretical analysis and PSIM simulation show that all aspects can achieve the desired results. The High-voltage power source system for EBM has the advantages of excellent control performance, high efficiency and good reliability.

\section{Acknowledgement}

This work is supported by Innovation Project of Guangxi Graduate Education, China(Grant No.YCSZ2015143). The corresponding author is Xuejiao Li, Guilin University of Electronic Technology, Emial:573351134@qq.com. 


\section{References}

[1] Shisha ZHU,He-ping HUANG and Quan BAI.Design and Simulation of a Electrorheological(ER) Control High Voltage Power Supply[J]. International Journal of Plant Engineering and Management, Vol.14(2009),No.03,p142-146.

[2] Jinhai MO, Shouqi WEI, Shaojia HE. Design of stabilized high -voltage source with PWM-BUCK for electron beam welder[J]. Transactions of the China Welding Institution, Vol.30(2009),No.06,p34-38. 